\title{
Superhydrophobic Materials With Good Oil/Water Separation And Self-Cleaning Prepared Through A Environment-Friendly And Two-Component Method
}

\section{Wensheng Lin}

Fujian Agriculture and Forestry University

\section{Mengting Cao}

Fujian Agriculture and Forestry University

Kehinde Olonisakin

Fujian Agriculture and Forestry University

Ran Li

Fujian Agriculture and Forestry University

Xinxiang Zhang ( $\nabla$ xxzhang0106@163.com )

Fujian Agriculture and Forestry University https://orcid.org/0000-0002-5343-6727

\section{Wenbin Yang}

Fujian Agriculture and Forestry University

\section{Research Article}

Keywords: Two-component, Superhydrophobic surface, Poly(methylhydrogen)siloxane (PMHS), Oil/water separation

Posted Date: June 8th, 2021

DOl: https://doi.org/10.21203/rs.3.rs-549413/v1

License: (c) (i) This work is licensed under a Creative Commons Attribution 4.0 International License. Read Full License 


\section{Abstract}

A novel, versatile, environment-friendly, and economical method was developed to fabricate functional superhydrophobic surfaces on various substrates, including wood, bamboo, cotton, filter paper, sponge, glass, textile, and copper. This method involves synthesizing a two-component modifier solution consisting of $\mathrm{SiO}_{2}$ nanoparticles combination with poly(methylhydrogen)siloxane (PMHS) modification.

The superhydrophobicity of the coated surfaces was created by PMHS combined with $\mathrm{SiO}_{2}$ nanoparticles to construct a rough hierarchical structure on the substrate surface. As a result, all superhydrophobic surfaces were maintained under an indoor environment and relative humidity (RH) of $50 \%$ for 30 days. Furthermore, the superhydrophobic surfaces were also maintained at environmental conditions of minus $20^{\circ} \mathrm{C}$ for 24 hours. It was also confirmed that these surfaces exhibited excellent self-cleaning, oil/water separation, and elimination of underwater oil properties. The method for fabricating superhydrophobic materials proposed in this study will have great application potential in preparing large-scale superhydrophobic surfaces for use in ancient building protection.

\section{Introduction}

Cellulose-based and metal materials are easy to absorb water and moisture. Therefore, their service life is dramatically affected, which leads to a serious waste of resources. Wood plays a vital role in modern society. Its numerous advantages (light weight, high strength, easy machinability) have been widely used in varied daily applications such as indoor and outdoor decoration, wooden bridge, pavement and so on (Lu et al. 2014; Poaty et al. 2013). However, due to cellulose and hemicelluloses' hydrophilic property, wood is sensitive to humidity changes, making it dimensionally unstable, susceptible to decay and degradation (Okon et al. 2018; Han et al. 2018). Like the inherent hydrophilic properties of wood, other cellulose-based materials such as paper, cotton, fabric, and bamboo commonly seen in daily human life also have problems such as easy degradation and decay (Miao et al. 2010; Hu et al. 2009). Nowadays, metal materials have been widely used in aerospace, energy, mechanical equipment, and other fields (Mikhail et al. 2016; Qiu et al. 2014). However, the corrosion resistance of metal materials is generally weak. It is easy to be affected by the humid environment, causing significant performance degradation and shortening its service life.

Consequently, there is an urgent need for effective protection of metals, cellulose-based, and other materials to enhance their corrosion resistance, durability, and water repellency, thereby extending their service life and expanding their application areas. Bionic construction of hydrophobic or superhydrophobic coatings on the surface of cellulose-based or metal materials is one of the most commonly used protection methods because these coatings can effectively reduce the damage caused by water/moisture to the material (Jia et al. 2016; Wu et al. 2016). The bionic construction of hydrophobic or superhydrophobic coating can significantly improve the water/moisture resistance and durability of metal and cellulose-based materials, especially in humid or outdoor conditions. 
During the past decades, superhydrophobic surfaces have gained remarkably increasing interest in material science because of their tremendous applications in anti-icing (Boinovich et al. 2016; Cao et al. 2009), anti-fogging (Lai et al. 2012; Chen et al. 2012), anti-corrosion (Cho et al. 2017; She et al. 2013), self-cleaning (Fürstner et al. 2005; Cho et al. 2017), water-oil separation (Zhang et al. 2013; Liu et al. 2018), and drag reduction (Daniello et al. 2009; Lee and Kim 2011), etc. Superhydrophobic surfaces are those with water contact angles (WCAs) larger than $150^{\circ}$ and sliding angles (SAs) below $10^{\circ}$ (Feng et al. 2002). Inspired by the lotus effect, a typical process to construct a superhydrophobic surface usually goes through two steps: (1) creation of a suitable roughness on material surfaces and (2) chemical modification of rough surfaces with low surface energy materials (Wang et al. 2009; Feng et al. 2002). Various approaches have been explored to fabricate superhydrophobic surfaces. For example, plasma treatment (Han and Moon 2015; Fernández-Blázquez et al. 2011), layer-by-layer assembly (Zhang et al. 2008), sol-gel processes (Wang et al. 2014; Wang et al. 2011; Taurino et al. 2008), chemical vapor deposition (CVD) (Ma et al. 2005; Zheng et al. 2009), hydrothermal treatments (Wang et al. 2012; Wu et al. 2015), and so on. However, these ways face serious industrialization challenges because of light opaqucity of the coating, requiring a tedious and time-consuming process, harsh conditions and specialized equipment, and environmental pollution (toxic solvent or modifier). For example, hydrothermal and plasma treatment are only applicable to the small surfaces and chemical vapor deposition only applies to special materials. Therefore, seeking an efficient, environment-friendly, lowcost, and scalable approach is the current research focus.

This work reports a simple, inexpensive, environment-friendly, but versatile method to fabricate superhydrophobic surfaces on cellulose-based and metal substrates. Specifically, a two-component modifier solution was efficiently synthesized using industrialized $\mathrm{SiO}_{2}$ nanoparticles, polymethylhydrosilane (PMHS), and ethanol as raw materials. The aim of the two-component package process is to isolate PMHS from Kastredt catalyst. Component A is composed of $\mathrm{SiO}_{2}, \mathrm{PMHS}_{\text {, ethanol, }}$ and inhibitor, while component $\mathrm{B}$ consists of $\mathrm{SiO}_{2}$, ethanol, and Kastredt catalyst. By simply dipping or spraying the two-component modifier solution (mixing A and B), superhydrophobic surfaces can be easily created on different substrates without the need for any pretreatment process. This two-component method has the following special features. (1) PMHS, and $\mathrm{SiO}_{2}$ nanoparticles (fumed $\mathrm{SiO}_{2}$ ) are massproduced products and therefore are easily handled and inexpensive. (2) There are no limits on the type or shape of substrates. Cellulose-based materials, glass, and metals can all be used without destroying their intrinsic appearance. (3) The superhydrophobic surfaces fabricated in this study showed dimensional stability, self-cleaning, and water/oil separation properties.

\section{Materials And Methods}

\section{Materials}

$\mathrm{SiO}_{2}$ nanoparticle (fumed silica, QS25) with a diameter of about $10 \mathrm{~nm}$ was purchased from Tokuyama Chemicals (Zhejiang) Co., Ltd. Ethanol (analytical grade) was purchased from Tianjin Zhiyuan Chemical 
Reagent Co., Ltd. PMHS with a content of active hydrogen (Si-H) of 1.5\% and Kastredt catalyst (platinum1,3-divinyl-1,1,3,3-tetramethyldisiloxane) with Pt content of 2000 ppm were purchased from Chengguang Research Institute of Chemical Industry (Chengdu, China). Inhibitor (1-ethynyl-Cyclohexanol) was purchased from Shenzhen OSBANG New Material Co., Ltd. The wood samples of Chinese Cunninghamia lanceolata for this study (obtained from Fujian, China) were cut parallel to grain direction and sawn into blocks of $20 \mathrm{~mm} \times 20 \mathrm{~mm} \times 12 \mathrm{~mm}$. Bamboo was produced by China Resources Bamboo Co., Ltd., Zhejiang, China. Cotton, sponge, life liquids, and other substrate were purchased from the native market. All chemicals in this work were used as received without further purification.

\section{Preparation of two-component modifier solution}

$\mathrm{SiO}_{2}$ nanoparticles $(0.5 \mathrm{~g})$ and PMHS $(1 \mathrm{~g})$ were dispersed in $39 \mathrm{~g}$ ethanol with the aid of ultrasonication for $20 \mathrm{~min}$. Inhibitor $(0.1 \mathrm{ml})$ was then added to the above ethanol dispersion to form the component $A$. $\mathrm{SiO}_{2}$ nanoparticles $(0.5 \mathrm{~g}$ ) were dispersed in $40 \mathrm{~g}$ ethanol through ultrasonication for $20 \mathrm{~min}$. Kastredt catalyst $(0.1 \mathrm{ml})$ was then added to the above ethanol dispersion to form the component B. Subsequently, the component $A$ and $B$ were mixed to form the two-component modifier solution.

\section{Fabrication of superhydrophobic surfaces on various substrates}

The superhydrophobic surfaces can be prepared on porous, nonporous, and homogeneous materials by simply brushing, spraying, or dipping into the two-component modifier solution. In this work, the various substrate (wood, bamboo, cotton, filter paper, sponge, glass, copper, textile) were dipped into the twocomponent modifier solution for 5 minutes, and then air-dried for 1 minute. This procedure was repeated for 3 times. Finally, the modified substrate was dried at $80^{\circ} \mathrm{C}$ for 1 hour.

\section{Characterization}

The surface morphology and microstructure of the samples were characterized using a scanning electron microscope (SEM, ZEISS Z500). Prior to SEM observation, the conductivity of the samples was improved by sputtering a thin layer of gold film on all samples. The static contact angle (CA) and sliding angle (SA) of water and other liquids (coffee, milk, soy sauce, juice, and beer) were measured on a commercial contact angle meter (HARKE-SPCA-1, Beijing) at room temperature. The volume of all liquids was $5 \mu \mathrm{l}$ when the contact angles were measured. The SA was measured by recording the tilt angle of the sample platform at which a droplet of liquids $(10 \mu \mathrm{l})$ starts to roll off the surface. The final CA and SA were calculated by averaging five different positions on each sample.

\section{Results And Discussion}

\section{Surface morphology and wettability}

SEM investigations allowed us to determine the microstructural morphology of substrates before and after surface modification. The morphology of the superhydrophobic surfaces of wood, glass, metal, and 
other cellulose-based materials is shown in Fig. 1. The original wood and bamboo surface was smooth and contained a few vessels. The unmodified filter paper and cotton was composed of smooth crosslinked fibers. The SEM image shows that the untreated glass and copper surfaces were smooth without any feature. On the contrary, after modification by the two-component modifier solution, almost all treated substrate surfaces possess a rough structure characterized by a random distribution of particles having various sizes and shapes.

Moreover, after coating, all treated substrate surfaces had a WCA greater than $150^{\circ}$ (spherical water droplets were standing on the treated substrate surfaces) and SA less than $10^{\circ}$ (as shown in Table 1). It was indicated that superhydrophobicity was successfully obtained. Based on the analysis mentioned above, it can be confirmed that the two-component modifier solution can create similar hierarchical structures with a high WCA on different types of substrates regardless of their morphologies or sizes.

Also, the treated substrate surfaces exhibited superhydrophobicity against water and against common liquids, including milk, coffee, juice, soy sauce, and beer. Figure 2 shows the relation between the liquids and liquid repellency of coatings on various substrates (The substrates included wood, bamboo, cotton, filter paper, sponge, glass, textile, and copper). The coatings' contact angles on different substrates were larger than $150^{\circ}$ for milk, coffee, juice, soy sauce, and beer. All the liquid droplets had a spherical shape and were well supported by the substrate surface treated with the two-component modifier solution, indicating good repellency towards these common liquids. The repellency towards common liquids implies the excellent anti-fouling potential of these superhydrophobic materials.

Table 1

Wettability of superhydrophobic surfaces on different substrates

\begin{tabular}{|lll|}
\hline Substrate & WCA $(\mathrm{deg})$ & SA $(\mathrm{deg})$ \\
\hline Wood & $154.1 \pm 2.1$ & $5.1 \pm 0.9$ \\
\hline Bamboo & $153.6 \pm 1.8$ & $5.8 \pm 1.3$ \\
\hline Cotton & $151.8 \pm 1.6$ & $8.2 \pm 0.5$ \\
\hline Filter paper & $154.7 \pm 2.5$ & $7.3 \pm 1.5$ \\
\hline Sponge & $151.3 \pm 0.9$ & $8.7 \pm 1.6$ \\
\hline Glass & $155.1 \pm 2.3$ & $4.0 \pm 0.8$ \\
\hline Copper & $152.6 \pm 1.6$ & $6.7 \pm 1.2$ \\
\hline Textile & $151.4 \pm 0.7$ & $8.4 \pm 1.5$ \\
\hline
\end{tabular}

\section{Durability of the coated superhydrophobic surfaces}

To be used in real life, the superhydrophobic surface should withstand a certain degree of damage. In this study, we conducted a comprehensive and systematic study on the prepared superhydrophobic surfaces' 
mechanical stability and durability.

In the past, most of the evaluation of the durability of superhydrophobic surfaces was carried out by immersing superhydrophobic materials in water (Lin et al. 2020). Nevertheless, few papers have explored the durability of superhydrophobic surfaces against moisture. Liquid water is larger than the pores formed in the rough structure, whereas moisture is small enough to penetrate the pores and attack the rough structure (Wu et al. 2016). In other words, the superhydrophobic surface only shows high hydrophobicity to liquid water, but cannot prevent the penetration of moisture. To assess the superhydrophobic surfaces' durability to moisture, eight types of superhydrophobic materials were investigated under an indoor environment, and a relative humidity $(\mathrm{RH})$ of $50 \%$ for 30 days. Figure 3 shows the variation of the WCA and SA in an indoor environment. The WCA and SA almost retained their original states, indicating long-term durability in an indoor environment. Therefore, these superhydrophobic surfaces on various substrates exhibit long-term durability in an indoor environment. Hence, these superhydrophobic materials can be well applied in indoor environments.

To evaluate the mechanical stability of the superhydrophobic surfaces, a sandpaper abrasion test was performed on the superhydrophobic surface according to the previously reported methods (Chang et al. 2015; Tu et al. 2018; Jia et al. 2018). As shown in Fig. 4, eight types of superhydrophobic materials were rubbed with sandpaper (1500 mesh) by loading $10 \mathrm{~g}$ weights on the sample. The WCA and SA value of the resultant surfaces were measured at every $20 \mathrm{~cm}$ of abrasion length. Figure 4 shows the waterrepellent ability of various superhydrophobic materials after $140 \mathrm{~cm}$ of abrasion. The results are as follows. Superhydrophobic wood after severe abrasion, remains superhydrophobic with a WCA greater than $150^{\circ}$ and an SA less than $10^{\circ}$, indicating excellent mechanical robustness against sandpaper abrasion. The good mechanical stability of the superhydrophobic wood surface can be attributed to the sufficient thickness of the superhydrophobic coating to withstand the abrasion and the hydroxyl group on the surface of the wood, which forms a covalent bond with the -Si-H on the PMHS chains. Similar to wood substrates, the superhydrophobic coating on the surface of bamboo also had good mechanical stability. However, superhydrophobic materials, including cotton, filter paper, sponge, glass, textile and copper, could not maintain their original superhydrophobicity with decreased CAs of $\sim 115^{\circ}$ and SAs of $\sim$ $60^{\circ}$, which may result from their low specific surface area and inability to form a covalent bond.

Apart from the sandpaper abrasion test, water immersion test and anti-freezing experiments were also conducted on superhydrophobic materials. Taking wood as the test sample, a anti-freezing test and water immersion test were carried out to further assess superhydrophobic surfaces (as shown in Fig. 5a-b). Briefly, Place the superhydrophobic wood in a low-temperature (minus $20^{\circ} \mathrm{C}$ ) environment or immersion the superhydrophobic wood in water for a certain period of time ( $0 \sim 24$ hours) to characterize the change in hydrophobicity. WCA and SA were measured every 3 hours. Figure 5a-b shows the variation of the WCA and SA after water immersion and low-temperature treatment. After 24 hours of water immersion and freezing, the WCA and SA almost retained their original states, indicating long-term durability under water or low-temperature environments. In addition, because many superhydrophobic surfaces easily lose their superhydrophobic properties in hot water (Li et al. 2016), water droplets with different temperature were 
used to study the hot water superhydrophobicity of the as-prepared surface (Fig. 5c). It can be clearly seen that even if water drops with a temperature close to $100{ }^{\circ} \mathrm{C}$ are dropped on the surface of the modified wood, it still has good water-repellency, and the water drops are spherical. In short, these superhydrophobic materials can be well applied in extreme environments.

\section{The investigation of formation mechanism}

Superhydrophobic surfaces were successfully synthesized via a novel method proposed in this paper. The formation mechanism of the superhydrophobic surface is described herein (illustration shown in Fig. 6). Firstly, a two-component modifier solution (A, B) was prepared and mixed to obtain the modifier solution. Then, the eight types of materials were immersed in the mixed solution for a certain period of time. Because the hydrophobic modifier PMHS (the PMHS chain contains a large quantity of reactive Si-H bonds and hydrophobic $-\mathrm{CH}_{3}$ groups) used in this experiment is easy to dehydrogenate with hydroxylcontaining substances in the presence of a catalyst, Kastredt catalyst was used. (Kastredt catalyst is the complex compound of platinum and 1,3-divinyl-1,1,3,3-tetramethyldisiloxane, which has very high catalytic activity for dehydrogenation between -Si-H bands of PMHS and hydroxyl groups of substances at room temperature) (Lin et al. 2018; Lin et al. 2020). Wood, bamboo, cotton, filter paper, $\mathrm{SiO}_{2}$, etc. all contain a large number of hydroxyl groups, so It can combine with PMHS to improve their hydrophobic properties. However, because the dispersant (absolute ethanol) used in the experiment also contains hydroxyl groups, it will also react with the hydrophobic modifier PMHS, which affects the reaction between $\mathrm{PMHS}$ and $\mathrm{SiO}_{2}$ and the base material, which will lead to the modifier solution failure. Therefore, an inhibitor was introduced in the experiment, which can well avoid the modifier solution's failure. As shown in Fig. 6, when the substrates are dried, the reaction between $\mathrm{PMHS}$ and $\mathrm{SiO}_{2}$, substrates, and the dispersant absolute ethanol is delayed due to the inhibitor's presence (as a ligand, the inhibitor will coordinate with the platinum atom in the catalyst and hinder the contact between the platinum atom and the reactant). During the drying process, the absolute ethanol gradually evaporates. After the absolute ethanol was completely evaporated and the inhibitor no longer had an inhibitory effect, the PMHS on the substrate surface reacted with $\mathrm{SiO}_{2}$ or the substrate to form a rough hierarchical structure (low surface energy materials combined with rough nanomaterials). Therefore, a superhydrophobic coating can be constructed on the surface of various substrates.

\section{The application of superhydrophobic surfaces}

Self-cleaning. Superhydrophobic surfaces with low adhesion to water will have good self-cleaning properties (Zhou et al. 2016). Water has a very low adhesion force on the superhydrophobic surface. The water droplets rolling on the superhydrophobic surface can take away the contaminants accumulated on the surface, mimicking the lotus leaf effect. Therefore, it can be considered that the contaminants accumulated on the superhydrophobic surface can be easily removed. Figure 7 shows the self-cleaning effect of filter paper and wood surface without and with superhydrophobic coating. Both filter paper and wood surfaces were contaminated by methyl blue powder. For modified filter paper and wood surface, as the water was dropping to the surface, the water droplets readily rolled off, removing the methyl blue 
powder and resulting in a dry and clean surface (Fig. 7( $a_{5}-a_{8}$ and $\left.\left.b_{5}-b_{8}\right)\right)$. On the contrary, water droplets merged with the methyl blue powder and stuck to the original filter paper and wood surface, leading to a wet and polluted surface (Fig. 7( $a_{1}-a_{4}$ and $\left.\left.b_{1}-b_{4}\right)\right)$. As shown in Movie S1 and Movie S2, methyl blue powder on the superhydrophobic filter paper and the wood surface can be rapidly and completely removed by spraying water onto it. In contrast, the methyl blue powder cannot be completely removed from the surface of original filter paper and wood.

Oil-water separation. The efficient separation of oil-water mixtures is a long-term problem and challenging environmental pollution such as crude oil leakage in the ocean from industrial production (Wu et al. 2016). Materials with special wettability, including superhydrophobic and superoleophilic properties, have been widely used in oil-water separations (Xue et al. 2011). Herein, the modified filter paper demonstrated superhydrophobic-super-oleophilic properties. So it was used to demonstrate potential applications for oil-water separation. As shown in Fig. 8, the oil-water separation experiment was carried out using filter equipment and superhydrophobic filter paper serving as filter elements. When the mixture of diesel and water is poured onto the superhydrophobic filter paper, the diesel rapidly diffuses and penetrates through the filter paper and falls into the beaker below. On the contrary, the water remains on the filter paper. In this manner, the effective separation of oil-water mixture was achieved. The separation efficiency was higher than $97 \%$.

Finally, through a 20 cycles of oil-water separation test, the modified filter paper still has good oil-water separation performance, which also shows good durability. It also means that the as-prepared filtration material can be repeatedly used to separate oil-water mixtures.

Elimination of underwater oil. Herein, the superhydrophobic cotton was assessed for the elimination of underwater oil droplet. Figure 9 shows an oil red-dyed $\mathrm{CCl}_{4}$ droplet resting at the bottom of the water. When the superhydrophobic cotton was brought into contact with the water, its whole surface turned to silver-like. Furthermore, once the modified cotton contacted the oil droplet, part of the $\mathrm{CCl}_{4}$ was sucked into the cotton due to the oleophilic effect. The longer the contact, the more oil is loaded. Finally, when the cotton was taken out of the beaker, the water was cleaned up with no trace of dyed oil. Consequently, it can be concluded that superhydrophobic materials can be well applied in the field of oil-water separation, which can effectively solve the environmental pollution caused by oil-water mixing and the problem of oilcontaminated water in industrial production.

\section{Conclusions}

In summary, a novel, environment-friendly, and versatile method to fabricate superhydrophobic surfaces using inorganic nanoparticles $\mathrm{SiO}_{2}$ combined with $\mathrm{PMHS}$ was developed. It was confirmed that the superhydrophobic surfaces were fabricated on a variety of substrates, including wood, bamboo, cotton, filter paper, sponge, glass, textile, and copper. All superhydrophobic surfaces were endowed with a high WCA larger than $150^{\circ}$ and a low water SA less than $10^{\circ}$. The superhydrophobicity originated from its rough and hierarchical structures formed by $\mathrm{SiO}_{2}$ combined with PMHS. In addition, these 
superhydrophobic surfaces exhibited good long-term durability, self-cleaning, oil/water separation properties, and elimination of underwater oil. Because this modification method can easily be used to prepare multifunctional superhydrophobic materials, it will have a good application prospect in the preparation of bionic materials.

\section{Declarations}

\section{Acknowledgments}

The authors gratefully acknowledge the support from the Leading Project of Fujian Province of China $(2019$ H0008) and the National Natural Science Foundation of China (61505029).

\section{References}

1. Boinovich LB, Emelyanenko AM, Emelyanenko KA, Maslakov KI (2016) Anti-icing properties of a superhydrophobic surface in a salt environment: an unexpected increase in freezing delay times for weak brine droplets. Phys Chem Chem Phys 18(4):3131-3136

2. Cao L, Jones AK, Sikka VK, Wu J, Gao D (2009) Anti-lcing Superhydrophobic Coatings. Langmuir 25(21):12444-12448

3. Chang H, Tu K, Wang X, Liu J (2015) Fabrication of mechanically durable superhydrophobic wood surfaces using polydimethylsiloxane and silica nanoparticles. RSC Adv 5(39):30647-30653

4. Chen Y, Zhang Y, Shi L, Li J, Xin Y, Yang T, Guo Z (2012) Transparent superhydrophobic/superhydrophilic coatings for self-cleaning and anti-fogging. Appl Phys Lett 101(3):033701-033707

5. Cho EC, Changjian CW, Chen HC, Chuang KS, Zheng JH, Hsiao YS, Lee KC, Huang JH (2017) Robust multifunctional superhydrophobic coatings with enhanced water/oil separation, self-cleaning, anticorrosion, and anti-biological adhesion. Chem Eng J 314:347-357

6. Daniello RJ, Waterhouse NE, Rothstein JP (2009) Drag reduction in turbulent flows over superhydrophobic surfaces. Phys Fluids 21(8):625-634

7. Fürstner R, Barthlott W, Neinhuis C, Walzel P (2005) Wetting and Self-Cleaning Properties of Artificial Superhydrophobic Surfaces. Langmuir 21(3):956-961

8. Feng L, Li S, Li Y, Li H, Zhang L, Zhai J, Song Y, Liu B, Jiang L, Zhu D (2002) Super-Hydrophobic Surfaces: From Natural to Artificial. Adv Mater 14(24):1857-1860

9. Fernández-Blázquez JP, Fell D, Bonaccurso E, Campo AD (2011) Superhydrophilic and superhydrophobic nanostructured surfaces via plasma treatment. J Colloid Interface Sci 357(1):234-238

10. Han D, Moon SY (2015) Development of superhydrophobic surface on glass substrate by multi-step atmospheric pressure plasma treatment. Thin Solid Films 587(31):34-38 
11. Han X, Yin Y, Zhang Q, Li R, Pu J (2018) Improved wood properties via two-step grafting with itaconic acid (IA) and nano-SiO ${ }_{2}$. Holzforschung 72(6):499-506

12. Hu Z, Zen X, Gong J, Deng Y (2009) Water resistance improvement of paper by superhydrophobic modification with microsized $\mathrm{CaCO}_{3}$ and fatty acid coating. Colloids Surf A 351(1-3):65-70

13. Jia S, Chen H, Luo S, Qing Y, Deng S, Yan N, Wu Y (2018) One-step approach to prepare superhydrophobic wood with enhanced mechanical and chemical durability: Driving of alkali. Appl Surf Sci 455:115-122

14. Jia S, Liu M, Wu Y, Luo S, Qing Y, Chen H (2016) Facile and scalable preparation of highly wearresistance superhydrophobic surface on wood substrates using silica nanoparticles modified by VTES. Appl Surf Sci 386:115-124

15. Lai Y, Tang Y, Gong J, Gong D, Chi L, Lin C, Chen Z (2012) Transparent superhydrophobic/superhydrophilic $\mathrm{TiO}_{2}$-based coatings for self-cleaning and anti-fogging. $\mathrm{J}$ Mater Chem 22(15):7420-7426

16. Lee C, Kim CJ (2011) Underwater Restoration and Retention of Gases on Superhydrophobic Surfaces for Drag Reduction. Phys Rev Lett 106(1):014502-014506

17. Li J, Lu Y, Wu Z, Bao Y, Xiao R, Yu H, Chen Y (2016) Durable, self-cleaning and superhydrophobic bamboo timber surfaces based on $\mathrm{TiO}_{2}$ films combined with fluoroalkylsilane. Ceram Int 42(8):9621-9629

18. Lin W, Huang Y, Li J, Liu Z, Yang W, Li R, Chen H, Zhang X (2018) Preparation of highly hydrophobic and anti-fouling wood using poly(methylhydrogen)siloxane. Cellulose 25(12):7341-7353

19. Lin W, Zhang X, Cai Q, Yang W, Chen H (2020) Dehydrogenation-driven assembly of transparent and durable superhydrophobic ORMOSIL coatings on cellulose-based substrates. Cellulose 27(1):78057821

20. Liu Z, Yu J, Lin W, Yang W, Li R (2018) Facile method for the hydrophobic modification of filter paper for applications in water-oil separation. Surf Coat Tech 352:313-319

21. Lu Y, Feng M, Zhan H (2014) Preparation of $\mathrm{SiO}_{2}$-wood composites by an ultrasonic-assisted sol-gel technique. Cellulose 21(6):4393-4403

22. Ma M, Mao Y, Gupta M, Gleason KK, Rutledge GC (2005) Superhydrophobic Fabrics Produced by Electrospinning and Chemical Vapor Deposition. Macromolecules 38(23):9742-9748

23. Miao H, Bao F, Cheng L, Shi W (2010) Cotton fabric modification for imparting high water and oil repellency using perfluoroalkyl phosphate acrylate via $y$-ray-induced grafting. Radiat Phys Chem 79(7):786-790

24. Mikhail B, Vadim A, Igor K, Andrey S, Aleksey L, Maltseva T (2016) Usage of spatial lattice metal structures as roofing for mechanical equipment rooms of hydroelectric power stations. Matec Web of Conferences 73:01012-01018

25. Okon KE, Lin F, Lin X, Chen C, Chen Y, Huang B (2018) Modification of Chinese fir (Cunninghamia lanceolata L) wood by silicone oil heat treatment with micro-wave pretreatment. Eur J Wood Wood 
Prod 76(1):221-228

26. Poaty B, Riedl B, Blanchet P, Blanchard V, Stafford L (2013) Improved water repellency of black spruce wood surfaces after treatment in carbon tetrafluoride plasmas. Wood Sci Technol 47(2):411422

27. Qiu S, Xue M, Zhu G (2014) Metal-organic framework membranes: from synthesis to separation application. Chem Soc Rev 43(16):6116-6140

28. She Z, Li Q, Wang Z, Li L, Chen F, Zhou J (2013) Researching the fabrication of anticorrosion superhydrophobic surface on magnesium alloy and its mechanical stability and durability. Chem Eng J 228:415-424

29. Taurino R, Fabbri E, Messori M, Pilati F, Pospiech D, Synytska A (2008) Facile preparation of superhydrophobic coatings by sol-gel processes. J Colloid Inter Sci 325(1):149-156

30. Tu K, Wang X, Kong L, Guan H (2018) Facile preparation of mechanically durable, self-healing and multifunctional superhydrophobic surfaces on solid wood. Mater Design 140:30-36

31. Wang J, Chen H, Sui T, Li A, Chen D (2009) Investigation on hydrophobicity of lotus leaf: Experiment and theory. Plant Sci 176(5):687-695

32. Wang S, Liu C, Liu G, Zhang M, Li J, Wang C (2011) Fabrication of superhydrophobic wood surface by a sol-gel process. Appl Surf Sci 258(2):806-810

33. Wang S, Wang C, Liu C, Zhang M, Ma H, Li J (2012) Fabrication of superhydrophobic spherical-like a$\mathrm{FeOOH}$ films on the wood surface by a hydrothermal method. Colloids Surf A 403:29-34

34. Wang X, Liu S, Chang H, Liu J (2014) Sol-Gel Deposition of $\mathrm{TiO}_{2}$ Nanocoatings on Wood Surfaces with Enhanced Hydrophobicity and Photostability. Wood Fiber Sci 46(1):109-117

35. Wu Y, Qing Y, Liang J, Liu M, Luo S (2015) Facile fabrication of superhydrophobic surfaces on wood substrates via a one-step hydrothermal process. Appl Surf Sci 330:332-338

36. Wu Y, Jia S, Qing Y, Luo S, Liu M (2016) A versatile and efficient method to fabricate durable superhydrophobic surfaces on wood, lignocellulosic fiber, glass, and metal substrates. J Mater Chem 4(37):14111-14121

37. Xue Z, Wang S, Lin L, Chen L, Liu M, Feng L, Jiang L (2011) A novel superhydrophilic and underwater superoleophobic hydrogel-coated mesh for oil/water separation. Adv Mater 23(37):4270-4273

38. Zhang C, Zhang S, Gao P, Ma H, Wei Q (2014) Superhydrophobic hybrid films prepared from silica nanoparticles and ionic liquids via layer-by-layer self-assembly. Thin Solid Films 570:27-32

39. Zhang L, Li Y, Sun J, Shen J (2008) Layer-by-layer fabrication of broad-band superhydrophobic antireflection coatings in near-infrared region. J Colloid Inter Sci 319(1):302-308

40. Zhang W, Shi Z, Zhang F, Liu X, Jin J, Jiang L (2013) Superhydrophobic and Superoleophilic PVDF Membranes for Effective Separation of Water-in-Oil Emulsions with High Flux. Adv Mater 25(14):2071-2076

41. Zheng Z, Gu Z, Huo R, Ye Y (2009) Superhydrophobicity of polyvinylidene fluoride membrane fabricated by chemical vapor deposition from solution. Appl Surf Sci 255(16):7263-7267 
42. Zhou L, Xu S, Zhang G, Cai D, Wu Z (2016) A facile approach to fabricate self-cleaning paint. Appl Clay Sci 132-133:290-295

\section{Figures}

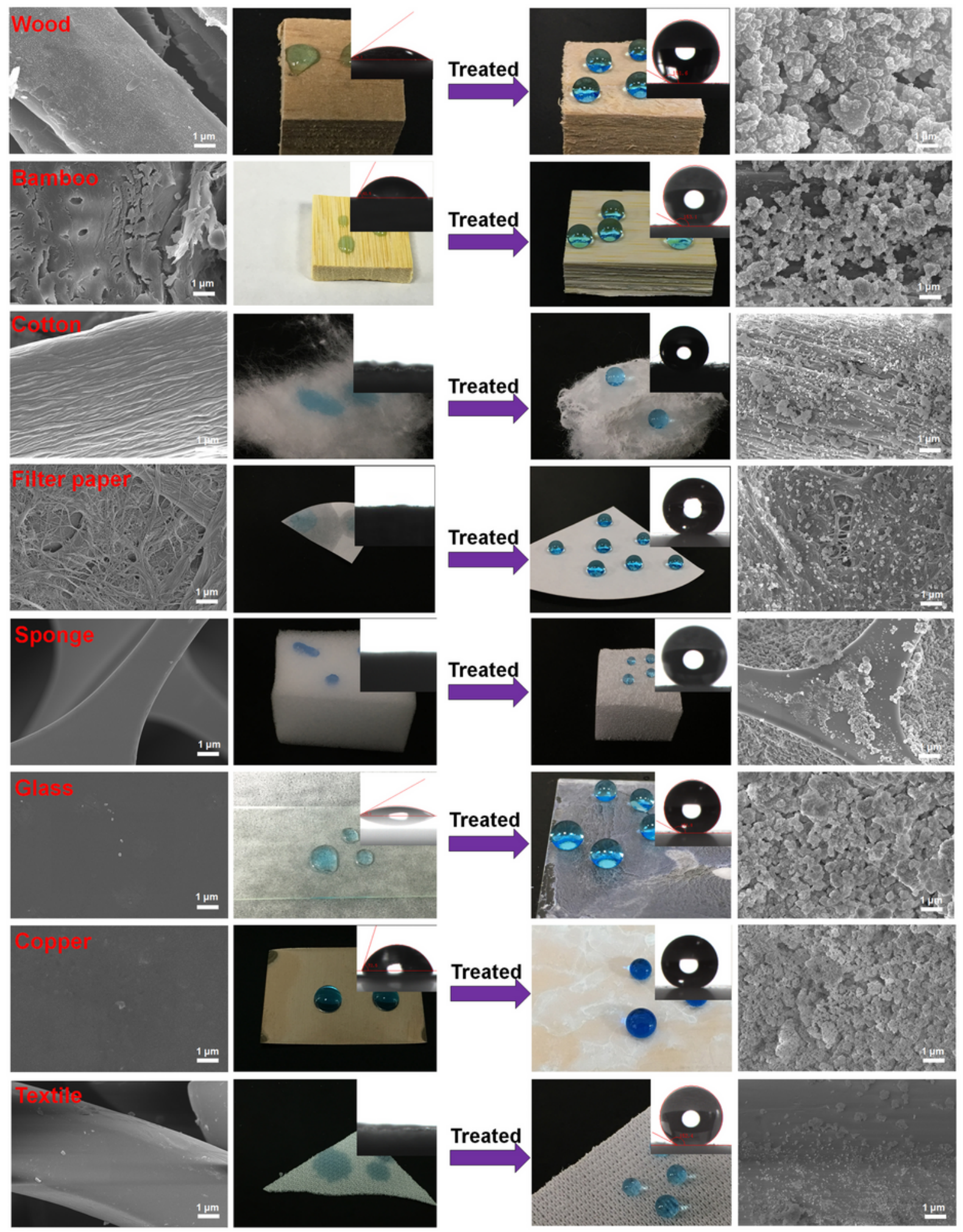

Figure 1 
Surface morphology of original and treated samples on various substrates.

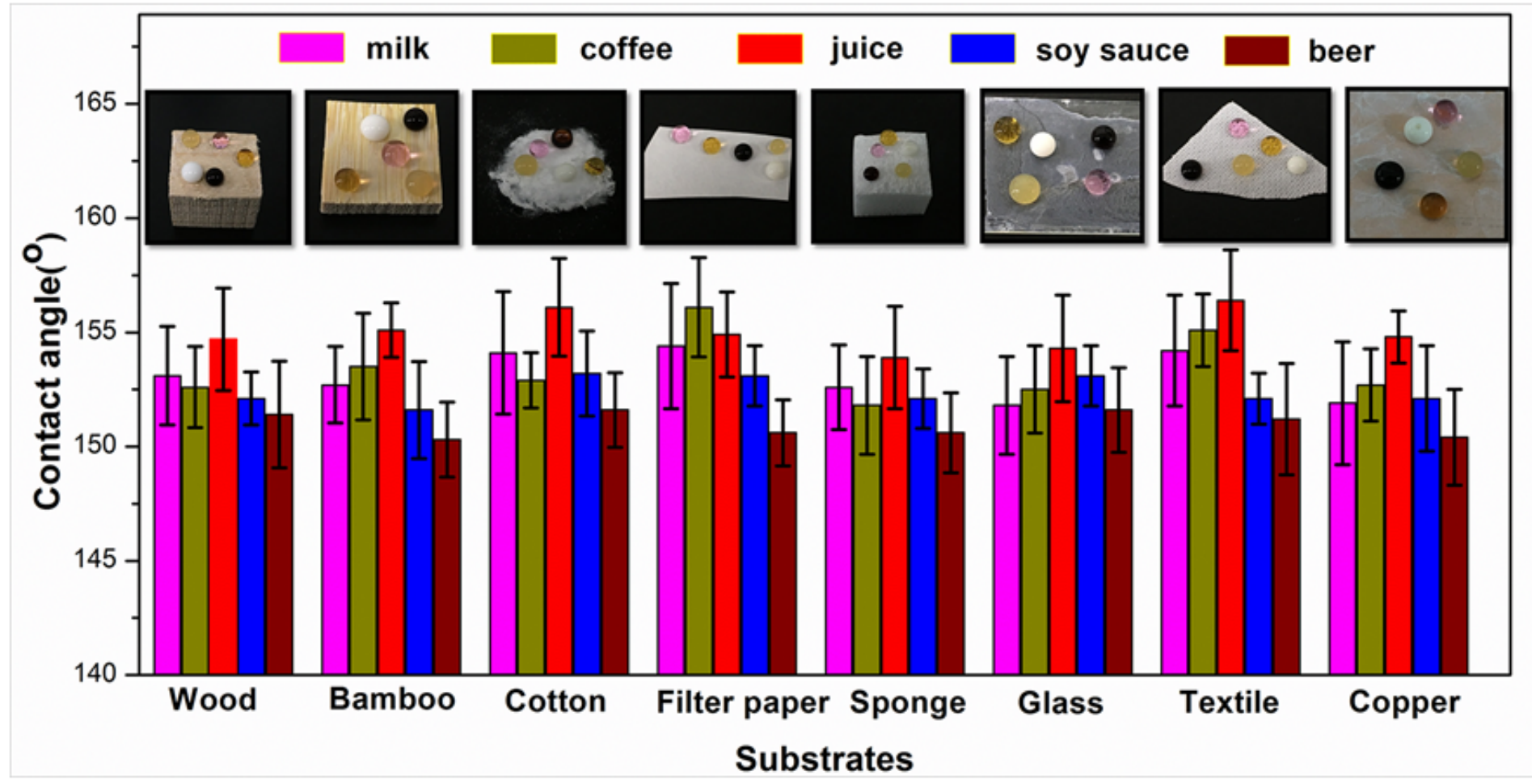

Figure 2

Wettability behavior of superhydrophobic surfaces exposed to milk, coffee, juice, soy sauce, and beer. 

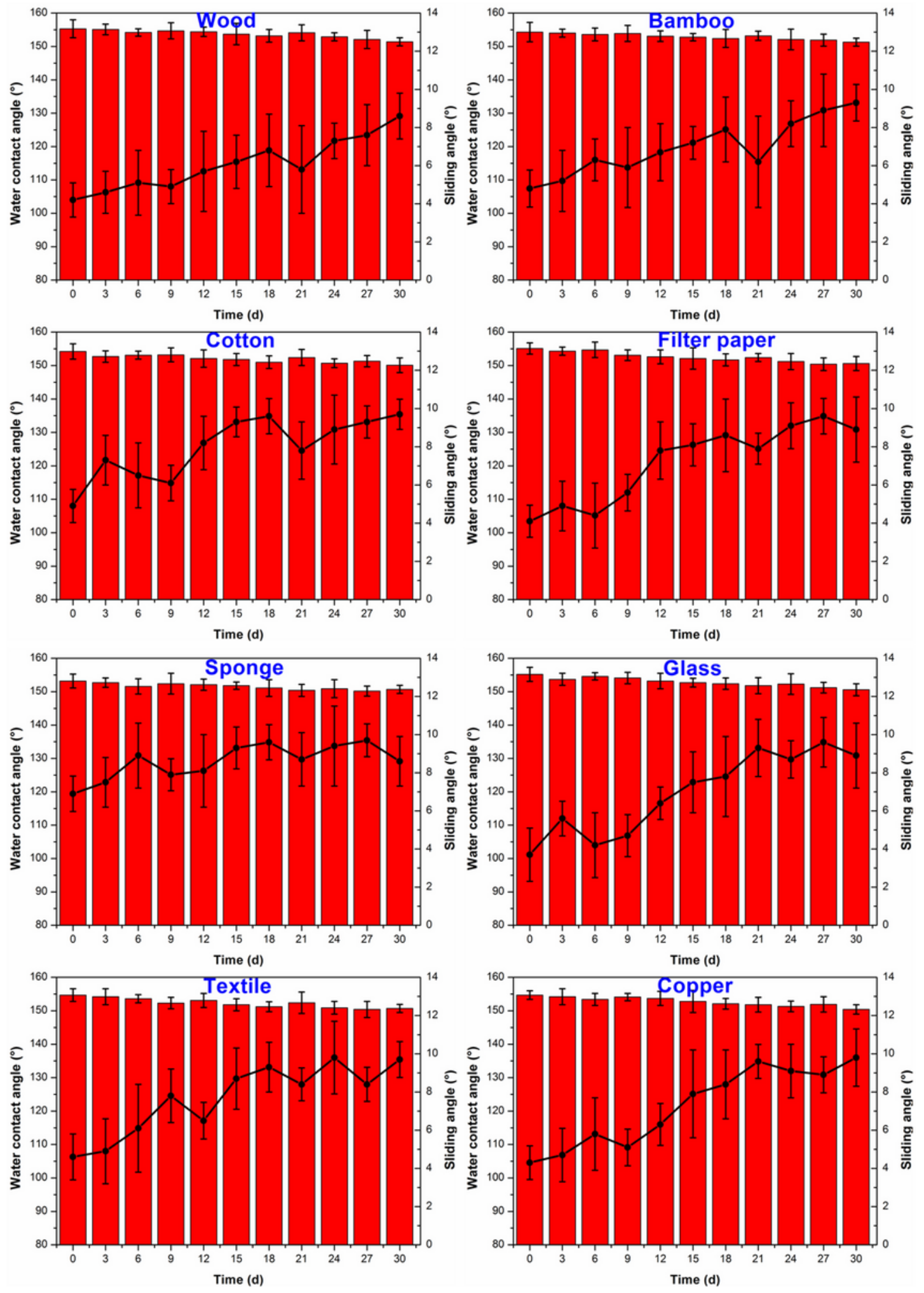

Figure 3

Variation of the WCA and SA under indoor environment . 
$10 \mathrm{~g}$

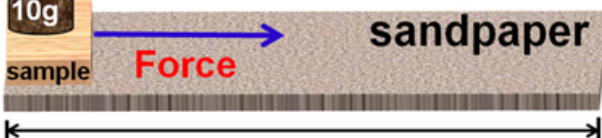

$20 \mathrm{~cm}$
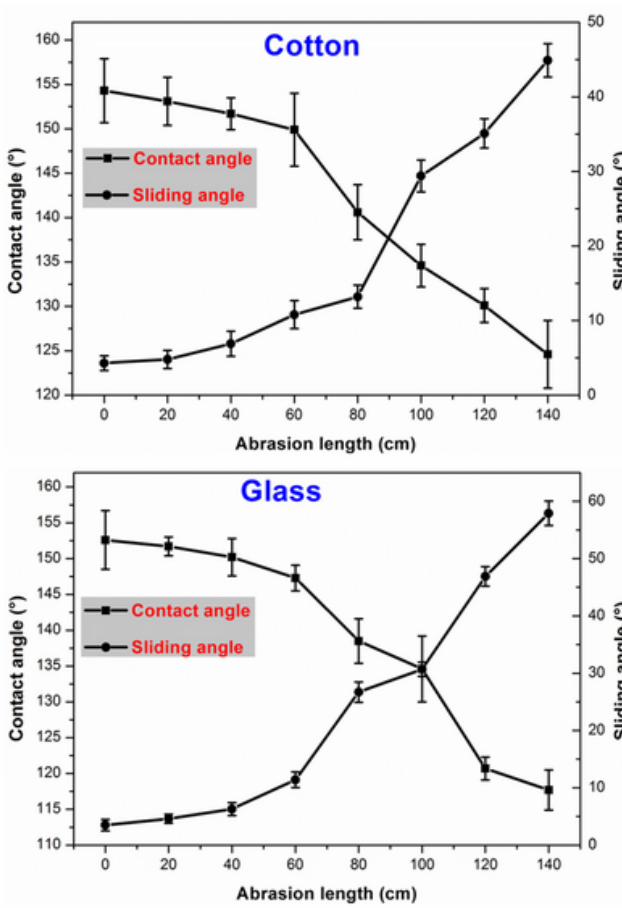
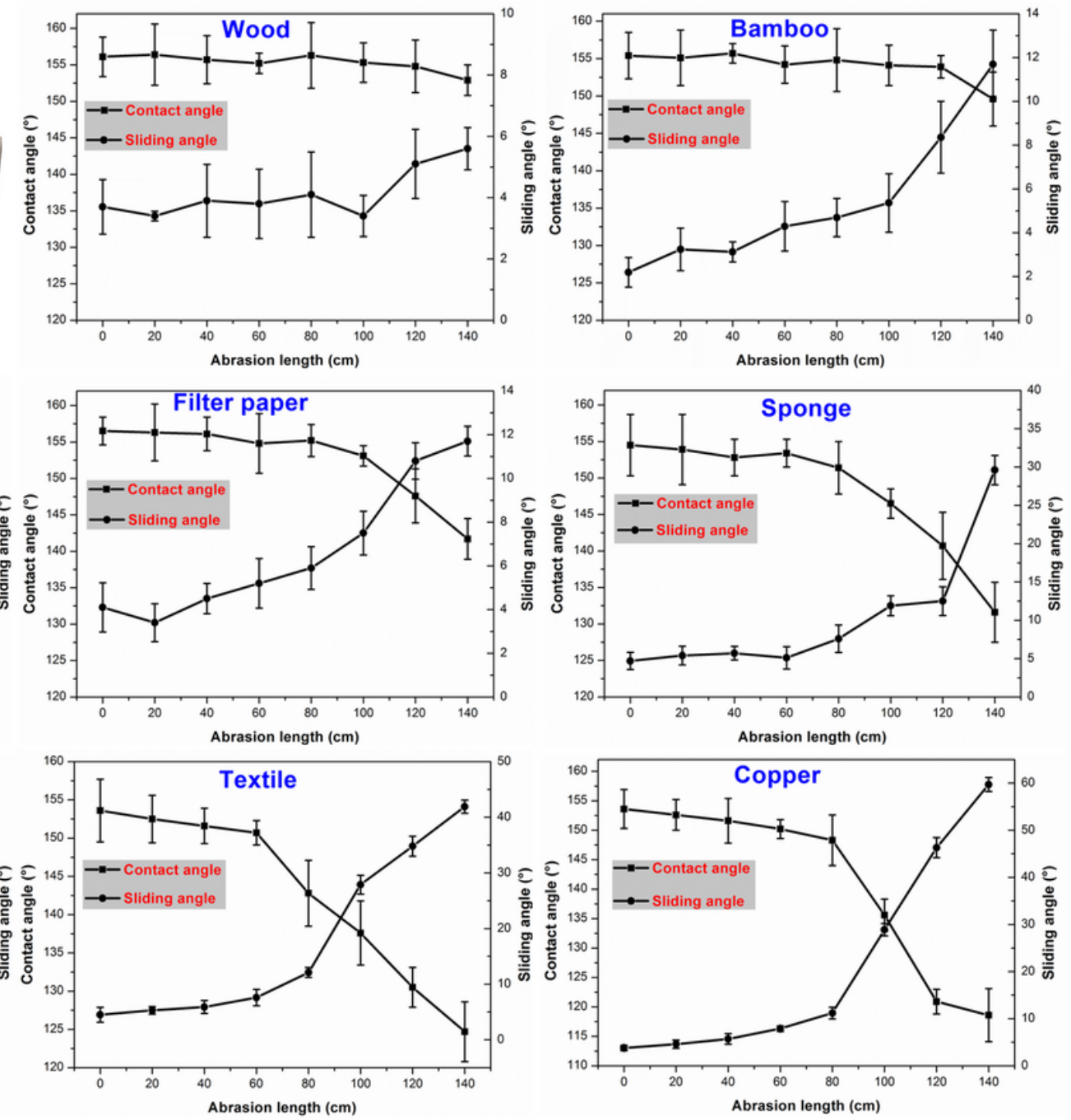

Figure 4

Schematic illustration of the abrasion test (the abrasion tests were carried out using SiC sandpaper (1500 mesh) as an abrasion surface) and the contact angle and sliding angle of superhydrophobic surfaces as a function of the abrasion length. 

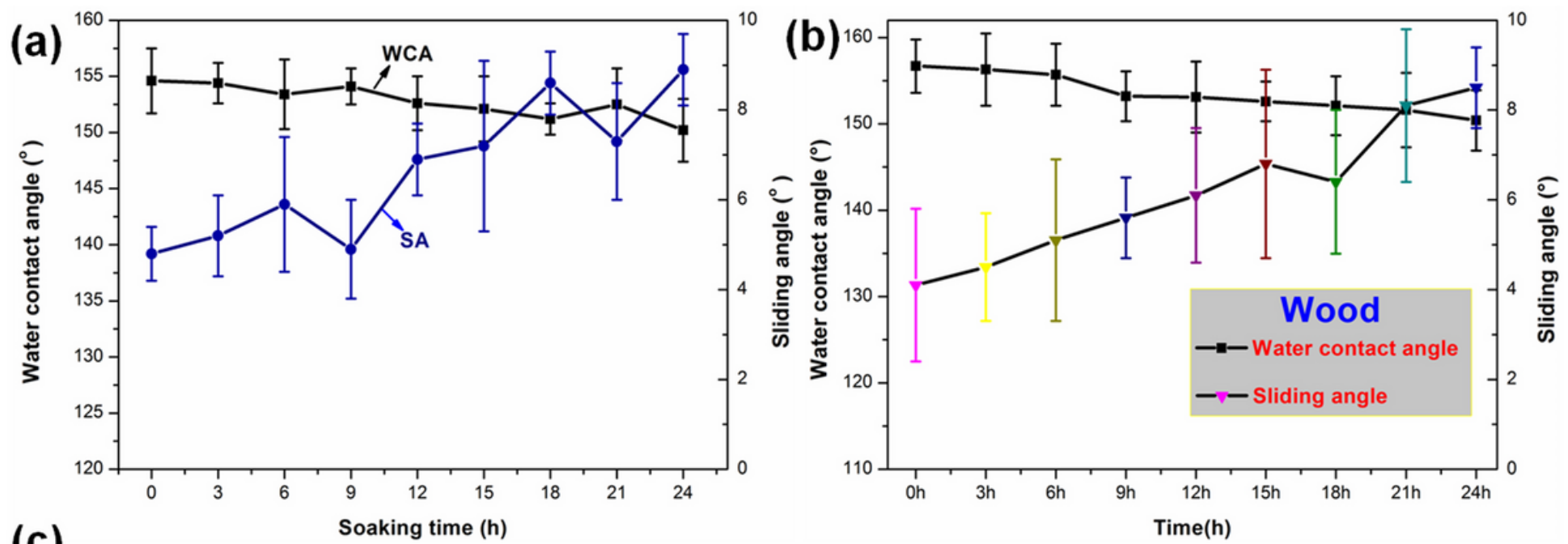

(c)
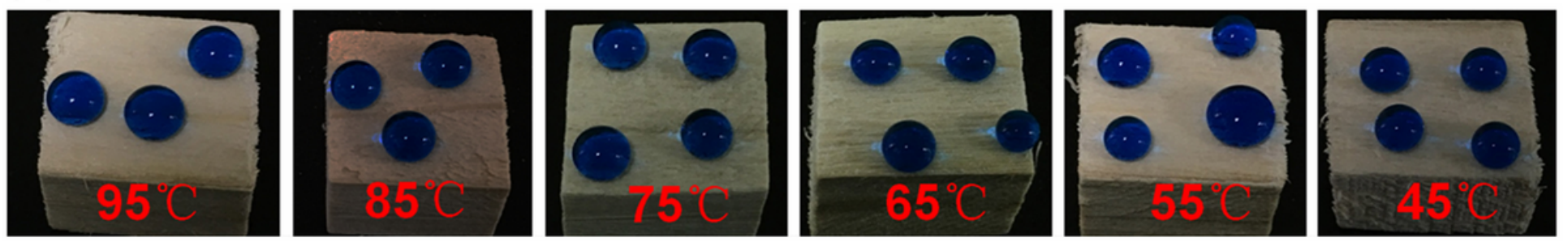

Figure 5

(a) Water immersion test; (b) Variation of the water contact angle and sliding angle in a low-temperature environment; (c) The photos of water droplets at different temperatures standing on the superhydrophobic wood. 


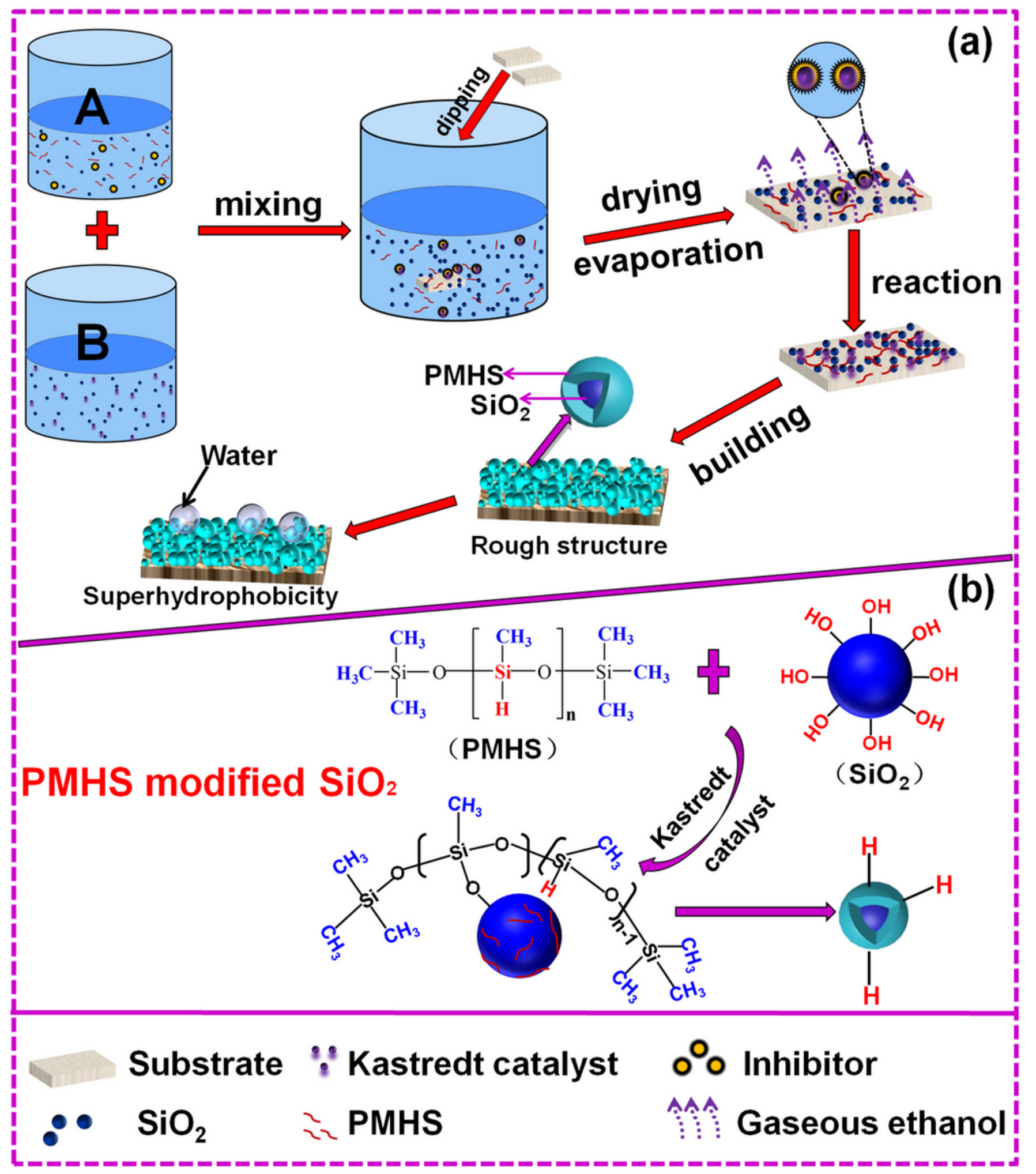

Figure 6

Illustration of the formation mechanism from hydrophilic to superhydrophobic states on various substrates. 

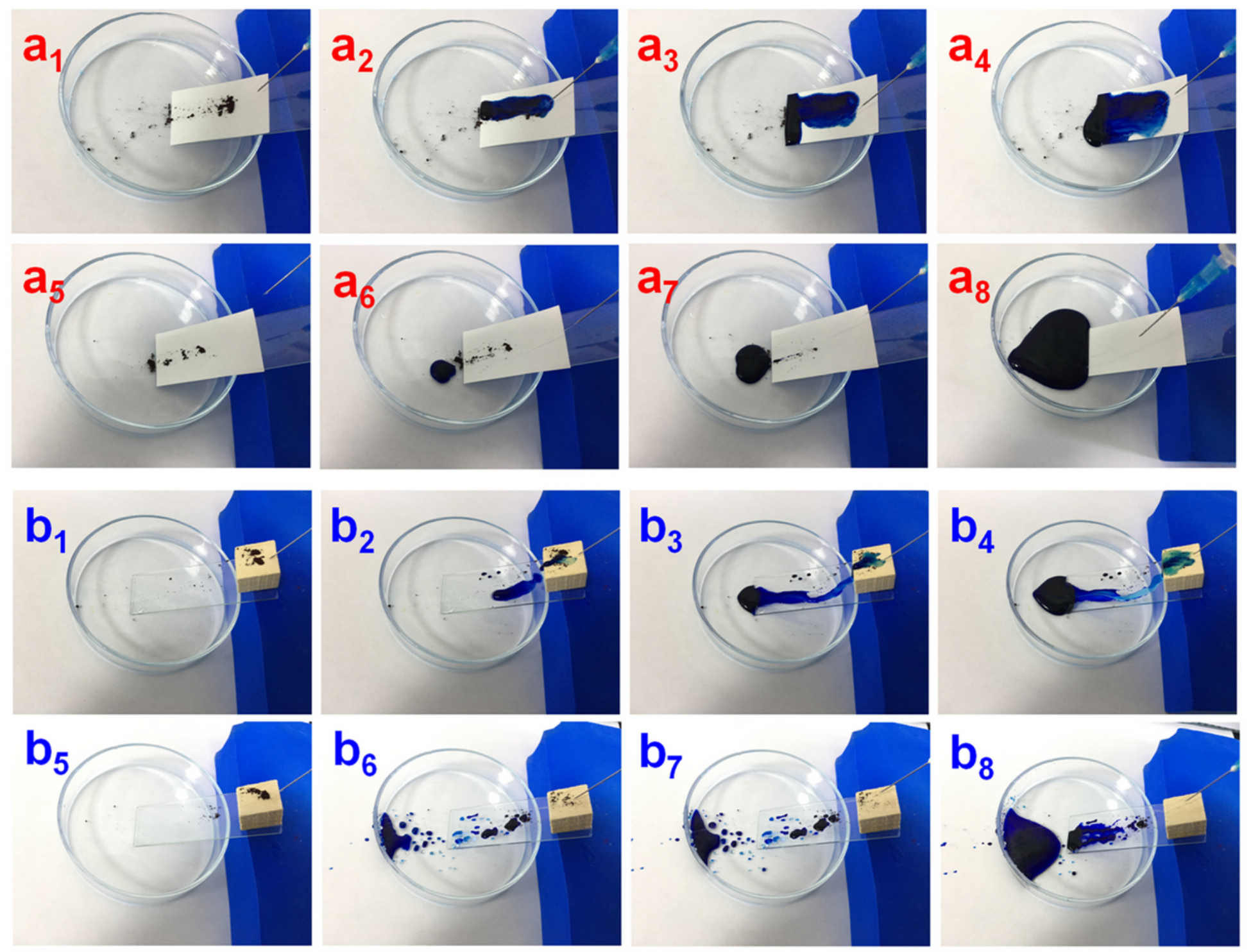

Figure 7

The evolution process of the self-cleaning behavior of the superhydrophobic surface coated on filter paper (a1-a4: untreated filter paper, a5-a8: treated filter paper) and wood (b1-b4: unmodified wood, b5-b8: modified wood). 
(a)
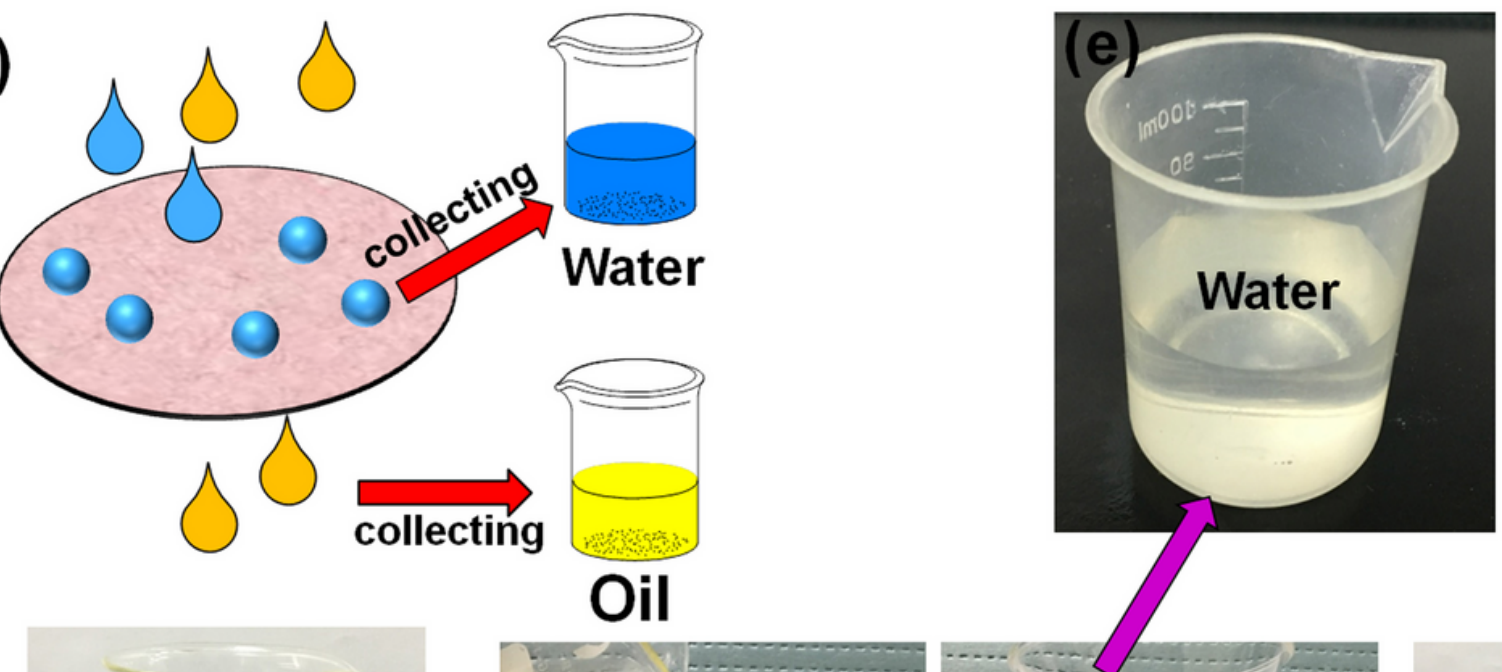

(b)

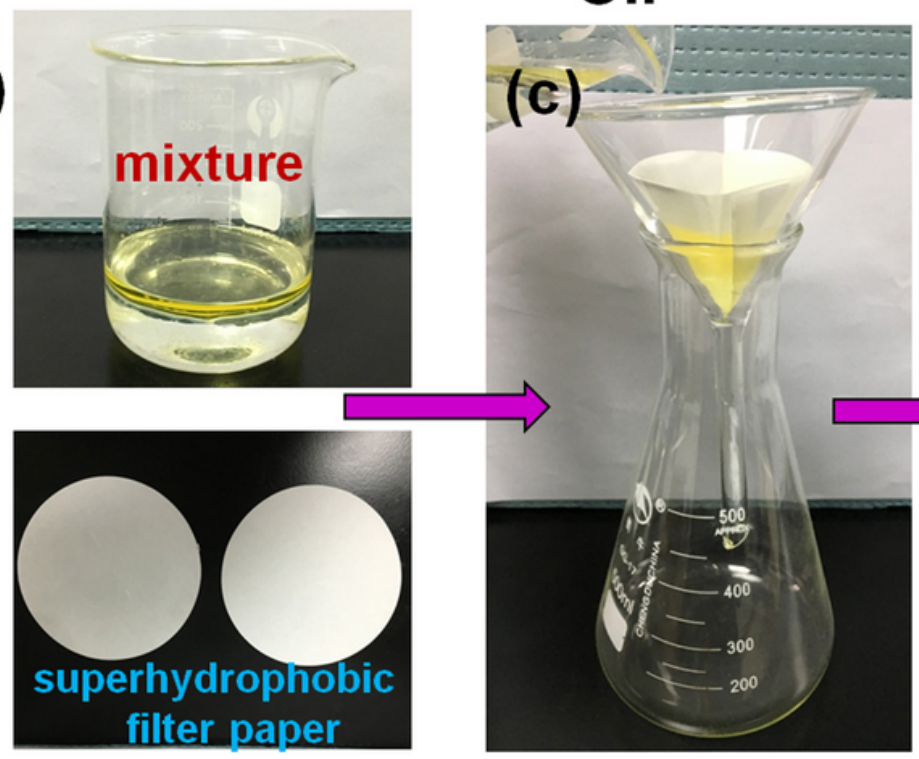

(d)

(f)

\section{Oil}

Figure 8

Schematic of the superhydrophobic material used to separate an oil-water mixture.

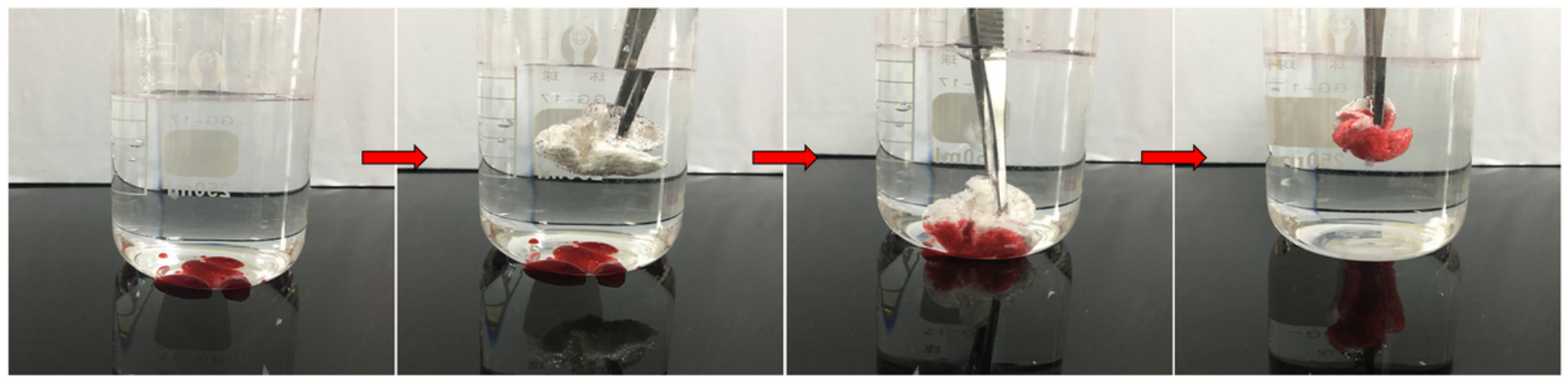

Figure 9

Elimination of underwater oil droplet (CCl4) by superhydrophobic cotton; the $\mathrm{CCl} 4$ is dyed red.

\section{Supplementary Files}


This is a list of supplementary files associated with this preprint. Click to download.

- GraphicalAbstract.jpg

- MovieS1.mp4

- Movies2.mp4 